\title{
Construction of automorphic Galois representations, II
}

\author{
Gaëtan Chenevier* and Michael Harris \\ In memory of Jon Rogawski
}

\section{Introduction}

Let $F$ be a totally real field, $\mathcal{K} / F$ a totally imaginary quadratic extension, $d=[F: \mathbb{Q}], c \in \operatorname{Gal}(\mathcal{K} / F)$ the non-trivial Galois automorphism. Let $n$ be a positive integer and $\mathcal{G}=\mathcal{G}_{n}$ be the algebraic group $R_{\mathcal{K} / \mathbb{Q}} G L(n)_{\mathcal{K}}$. The purpose of this article is to prove the existence of a compatible family of $n$ dimensional $\lambda$-adic representations $\rho_{\lambda, \Pi}$ of the Galois group $\Gamma_{\mathcal{K}}=\operatorname{Gal}(\overline{\mathcal{K}} / \mathcal{K})$ attached to certain cuspidal automorphic representations $\Pi$ of $\mathcal{G}$. Here is the precise statement (see Theorem 3.2.3):

Theorem. Fix a prime $p$ and a pair of embeddings

$$
\iota=\left(\iota_{p}: \overline{\mathbb{Q}} \rightarrow \overline{\mathbb{Q}}_{p}, \iota_{\infty}: \overline{\mathbb{Q}} \rightarrow \mathbb{C}\right) .
$$

Let $\Pi$ be a cuspidal automorphic representation of $G L(n, \mathcal{K})$ that is cohomological and conjugate self-dual in the sense of Hypotheses 1.1 below. Then there exists a semisimple continuous Galois representation

$$
\rho_{\iota, \Pi}: \Gamma_{\mathcal{K}} \rightarrow G L\left(n, \overline{\mathbb{Q}}_{p}\right)
$$

associated to $\Pi$ in the following sense:

(a) For all finite primes $v$ of $\mathcal{K}$ of residue characteristic prime to $p$,

$$
\left(\left.\rho_{\iota, \Pi}\right|_{\Gamma_{v}}\right)^{F-s s} \prec \mathcal{L}\left(\Pi_{v} \otimes|\bullet| \frac{1-n}{v^{2}}\right),
$$

*Financé par le C.N.R.S., partiellement soutenu par l'Agence Nationale de la Recherche, "ArShiFo ANR-BLAN-0114". 
where $\Gamma_{v}$ is a decomposition group at $v, \mathcal{L}$ is the local Langlands correspondence, the superscript ${ }^{F-s s}$ denotes Frobenius semisimplification, and the relation $\prec$ is the partial ordering on the associated Weil-Deligne representations defined in [Ch] $\S 3.1$;

(b) For all finite primes $v$ of $\mathcal{K}$ of residue characteristic $p,\left.\rho_{\iota, \Pi}\right|_{\Gamma_{v}}$ is de Rham (in Fontaine's sense), and its Hodge-Tate numbers have multiplicity at most one (i.e., $\rho_{\iota, \Pi}$ is Hodge-Tate regular) and are determined by the archimedean component $\Pi_{\infty}$ of $\Pi$ in accordance with an explicit recipe.

(c) Let $v$ be a finite prime of $\mathcal{K}$ dividing $p$. Suppose $\Pi_{v}$ has a non-zero vector fixed by a maximal compact subgroup of $G L\left(n, \mathcal{K}_{v}\right)$. Then $\rho_{v}:=$ $\left.\rho_{\iota, \Pi}\right|_{\Gamma_{v}}$ is crystalline, and if $\varphi$ denotes the smallest linear power of the crystalline Frobenius of $D_{\text {crys }}\left(\rho_{v}\right)$ then

$$
\operatorname{det}\left(T-\varphi \mid D_{c r i s}\left(\rho_{v}\right)\right)=\operatorname{det}\left(T-\mathcal{L}\left(\Pi_{v} \otimes|\bullet| \frac{1-n}{v^{2}}\right)\left(F r o b_{v}\right)\right) \text {. }
$$

We also show that $\rho_{\iota, \Pi}$ is semistable, in Fontaine's sense, at places $v$ of characteristic $p$ where $\Pi_{v}$ has a non-zero Iwahori-fixed vector, and attach $\lambda$-adic representations of $\Gamma_{F}=\operatorname{Gal}(\bar{F} / F)$ to cuspidal automorphic representations of $R_{F / \mathbb{Q}} G L(n)_{F}$ satisfying analogous hypotheses. In the remainder of this introduction we write $\rho_{\lambda, \Pi}$ rather than $\rho_{\iota, \Pi}$, to emphasize that we are constructing compatible families in Serre's sense. Occasionally the varying rational prime will be denoted $\ell$ rather than $p$ in the main text.

The first version of the main theorem of this article was proved by Clozel in [C2], using the results of [K2]. The theorem of Clozel and Kottwitz required $\Pi$ to belong to the discrete series at one or more non-archimedean places; more precise versions of this theorem were proved in [HT] and [TY], under the same local hypothesis. These results were based on a version of stable base change relating automorphic representations of $\mathcal{G}$ to automorphic representations of certain twisted unitary groups over $F$.

It was long understood that the local hypothesis of $[\mathrm{C} 2, \mathrm{HT}]$ could be removed once a sufficiently general stable trace formula was available for more general unitary groups. For $n \leq 3$ this program was carried out by Blasius and Rogawski, using the stable trace formula developed by Rogawski in $[R]$. For $n=3$ the Galois representations are constructed in general in [BR1] in the cohomology of Shimura varieties (Picard modular surfaces) attached to quotients of the unit ball in $\mathbb{C}^{2}$, or alternatively to unitary groups over $F$ of signature $(1,2)$ at one real place and definite elsewhere. The Galois representations for $n=2$ are constructed in [BR2] in the cohomology of the same Shimura varieties, using endoscopic transfer from $U(2) \times U(1)$ to $U(3)$. 
The proof of these results in [BR2] revealed that not every $\Pi$ can be treated in this way. For example, when the degree $d$ of $F$ is even and $\Pi$ is obtained by base change from an everywhere unramified Hilbert modular newform $f$ for $F$ of parallel weight $(2, \ldots, 2)$, any endoscopic transfer of the corresponding representation of $U(2) \times U(1)$ to $U(3)$ gives rise to a 1-dimensional Galois representation (corresponding to the $U(1)$ factor). The missing Galois representations were constructed by Wiles [W] (for $\lambda$ dividing primes at which $\Pi$ is ordinary), using Hida families, and in general by Taylor $[\mathrm{T}]$, using pseudorepresentations and congruences between old and new Hilbert modular forms.

Unlike the representations constructed in [BR2], it is not known that those introduced in $[\mathrm{W}]$ and $[\mathrm{T}]$ are motivic in the sense of Grothendieck. It is expected, for example, that a Hilbert modular newform $f$ of parallel weight $(2, \ldots, 2)$ whose Hecke eigenvalues are in $\mathbb{Q}$ is attached to an elliptic curve over $F$. This is known when $d$ is odd or when the associated automorphic representation is supercuspidal or Steinberg at some finite place, but when $d$ is even and $f$ is everywhere unramified, it is not known how to construct the hypothetical elliptic curve. The question was raised for $d=2$ thirty years ago in $[\mathrm{O}]$ and remains open to this day.

When Laumon and Ngô proved the fundamental lemma for unitary groups it became clear that the Blasius-Rogawski method could be applied for general $n$. This was carried out in the setting of the book project [L.IV.1, CHL.IV.2, CHL.IV.3] and independently by Shin in [S]. Shin's results are more precise - in particular, he treats primes of bad reduction, generalizing the methods of [HT] and [TY], and appear to be the most complete possible in the setting of Shimura varieties. The results of $[\mathrm{S}]$ are completely general when $n$ is odd; for even $n$ the obstruction discovered by Blasius and Rogawski generalizes, and endoscopic transfer from $U(n) \times U(1)$ to $U(n+1)$ only yields $n$-dimensional Galois representations when $\Pi$ is what Shin calls "slightly regular" (also called "Shin-regular").

As for $n=2$, the missing Galois representations for general even $n$ can be constructed by $p$-adic approximation. This is the purpose of the present paper. We generalize the method of $[\mathrm{W}]$ rather than that of $[\mathrm{T}]$. The theory of eigenvarieties developed in $[\mathrm{BC}]$ and $[\mathrm{Ch}]$ (generalizing the construction of Coleman and Mazur for elliptic modular forms) makes the ordinary hypothesis of $[\mathrm{W}]$ superfluous. The stable trace formula methods of [L.IV.1] provide a descent from $\Pi$ to automorphic representations of a totally definite unitary group $G_{0}$, and thus $\Pi$ gives rise to a point $x=x(\Pi)$ on an appropriate eigenvariety. For almost all $\lambda$, the Galois representation $\rho_{\lambda, \Pi}$ is 
obtained by specializing the families of Galois representations (or pseudorepresentations) constructed in $[\mathrm{BC}]$ and $[\mathrm{Ch}]$ to $x(\Pi)$. These families are in turn obtained as $\lambda$-adic interpolations of the representations attached in $[\mathrm{S}]$ to slightly regular $\Pi$. (The article [CHL.IV.3] uses a more restrictive regularity condition but also suffices for the construction of families of pseudo-representations.)

Eigenvarieties only detect automorphic representations of finite slope; for a given $\Pi$ this excludes a finite set of $\lambda$. The remaining $\rho_{\lambda, \Pi}$ are constructed by a generalization of a patching argument first introduced in [BRa] and generalized in [HT]. The patching argument we use is due to Sorensen [So] (an improvement of a less precise version considered by one of the authors of the present paper) and allows us to use solvable base change to reduce to the case of finite slope.

It turns out that slightly regular $\Pi$ do not suffice for most applications to automorphy lifting theorems. In fact, the automorphic Galois representations most useful for deformation theory are precisely those that cannot be realized motivically, cf. for example [BGHT, BGGT1]. The results of the present paper were designed for these applications. In particular we prove a weak version of compatibility of the correspondence $\Pi \leftrightarrow \rho_{\lambda, \Pi}$ with the local Langlands correspondence at primes not dividing the residue characteristic $p$ of $\lambda$. The weak version of local-global compatibility proved as (a) in the theorem above actually suffices for some applications, e.g. for proving the non-vanishing of Selmer groups by Ribet's method (see e.g. [BC]). Complete local-global compatibility at primes not dividing $p$, generalizing the results of [TY], has since been proved by Caraiani [Ca] by generalizing the Rapoport-Zink spectral sequence for vanishing cycles.

We actually give two different proofs that $\rho_{\lambda, \Pi}$ is de Rham with the expected Hodge-Tate numbers at primes dividing $p$, and crystalline at places at which $\Pi$ is unramified. One relies on the study of certain Shimura varieties attached to unitary groups of signature $(2, n-2)$; the second, which leads to more complete results, relies on an application of $p$-adic families with partial Hodge-Tate weight fixed found in [Ch]. For primes dividing $p$, a (nearly) complete compatibility result is proved in [BGGT2].

This is a revised version of the articles "Construction of automorphic Galois representations I, II" by Michael Harris and of an earlier version of the present article. ${ }^{1}$ The original intention was to publish this article in the second volume of the projected series of books entitled "Stabilization

\footnotetext{
${ }^{1}$ Previously posted at http://www.institut.math.jussieu.fr/projets/fa/bp0.html and http://fa.institut.math.jussieu.fr/node/45, respectively.
} 
of the trace formula, Shimura varieties, and arithmetic applications." The first volume has now appeared, and we have used some of the chapters as references. In the meantime, the preliminary version of the present article has been cited by a number of authors, notably for the applications already mentioned to automorphy lifting theorems. It therefore seemed appropriate to provide a reliable source for the cited results.

The proofs follow an outline first presented by one of the authors at the eigenvariety semester at Harvard in 2006. We thank the organizers of that semester, and especially Barry Mazur, for providing the opportunity to do so. We are grateful to the participants in the Paris automorphic forms book project, especially Laurent Clozel, Jean-Pierre Labesse, and Claus Sorensen for their collaboration and for making their results available. We thank Joel Bellaïche, Don Blasius, Sug Woo Shin, Peter Scholze, and Richard Taylor for helpful discussions.

\section{Construction of Galois representations under simplifying hypotheses}

Notation is as in the introduction. Let $\mathfrak{g}=\operatorname{Lie}(\mathcal{G}(\mathbb{R})), K_{\infty} \subset \mathcal{G}(\mathbb{R})$ the product of a maximal compact subgroup with the center $Z_{\mathcal{G}}(\mathbb{R})$. We consider cuspidal automorphic representations $\Pi$ of $\mathcal{G}$ satisfying the following two hypotheses:

General Hypotheses 1.1. Writing $\Pi=\Pi_{\infty} \otimes \Pi_{f}$, where $\Pi_{\infty}$ is an admissible $\left(\mathfrak{g}, K_{\infty}\right)$-module, we have

(i) (Regularity) There is a finite-dimensional irreducible representation $W(\Pi)$ of $\mathcal{G}(\mathbb{R})$ such that

$$
H^{*}\left(\mathfrak{g}, K_{\infty} ; \Pi_{\infty} \otimes W(\Pi)\right) \neq 0 .
$$

(ii) (Polarization) The contragredient $\Pi^{\vee}$ of $\Pi$ satisfies

$$
\Pi^{\vee} \stackrel{\sim}{\longrightarrow} \Pi \circ c .
$$

We next make the following temporary hypotheses:

\section{Special Hypotheses 1.2.}

(1.2.1) $\mathcal{K} / F$ is unramified at all finite places (in particular $d>1$ ).

(1.2.2) $\Pi_{v}$ is spherical (unramified) at all non-split non-archimedean places $v$ of $\mathcal{K}$.

(1.2.3) The degree $d=[F: \mathbb{Q}]$ is even. 
The irreducible representation $W(\Pi)$ factors over the set $\Sigma$ of real embeddings of $F$

$$
W(\Pi)=\otimes_{\sigma \in \Sigma} W_{\sigma},
$$

where $W_{\sigma}$ is an irreducible representation of $G L\left(n, \mathcal{K} \otimes_{F, \sigma} \mathbb{C}\right) \stackrel{\sim}{\longrightarrow} G L(n, \mathbb{C}) \times$ $G L(n, \mathbb{C})$. The highest weight of $W_{\sigma}$ is denoted $\mu(\sigma)$. It can be identified in the usual way with a pair of non-increasing $n$-tuples of non-negative integers $\left(\mu(\tilde{\sigma}), \mu\left(\tilde{\sigma}^{c}\right)\right)$, one for each extension $\tilde{\sigma}$ of $\sigma$ to an embedding of $\mathcal{K}$, where we write

$$
\mu(\tilde{\sigma})=\left(\mu_{1}(\tilde{\sigma}) \geq \mu_{2}(\tilde{\sigma}) \geq \ldots \mu_{n}(\tilde{\sigma})\right) .
$$

Moreover, the polarization condition implies that one of the $n$-tuples diagrams is dual to the other, in other words that

$$
\mu_{i}\left(\tilde{\sigma}^{c}\right)=-\mu_{n-i+1}(\tilde{\sigma})
$$

Special Hypothesis 1.3. For at least one $\sigma \in \Sigma$, the highest weight $\mu(\sigma)$ is sufficiently far from the walls; in practice, it suffices to assume $\mu(\sigma)$ is regular, i.e. $\mu_{i}(\tilde{\sigma}) \neq \mu_{j}(\tilde{\sigma})$ if $i \neq j$.

Let $K$ be a $p$-adic field, $W D_{K}$ its Weil-Deligne group. Let $\mathcal{A}(n, K)$ denote the set of equivalence classes of irreducible admissible representations of $G L(n, K)$, and let $\mathcal{G}(n, K)$ denote the set of equivalence classes of $n$ dimensional Frobenius semisimple representations of $W D_{K}$. We denote by

$$
\mathcal{L}: \mathcal{A}(n, K) \rightarrow \mathcal{G}(n, K)
$$

the local Langlands correspondence, normalized to coincide with local class field theory when $n=1$ in such a way that a uniformizer of $K^{\times}$is sent to a geometric Frobenius.

The following result has now been proved in several stages (articles [L.IV.1, CHL.IV.2, CHL.IV.3] of Book 1, and especially [S], of which an expository account will appear in Book 2). When $v$ is a finite place of $\mathcal{K}$ we let $\Gamma_{v}$ denote a decomposition group at $v$.

Theorem 1.4. (i) Suppose $n$ is odd and $\Pi$ satisfies Hypotheses (1.1) and (1.2). Then there is a number field $E(\Pi)$ and a compatible system $\rho_{\lambda, \Pi}$ : $\Gamma_{\mathcal{K}} \rightarrow G L\left(n, E(\Pi)_{\lambda}\right)$ of semi-simple $\lambda$-adic representations, where $\lambda$ runs through the finite places of $E(\Pi)$, such that

(a) For all finite primes $v$ of $\mathcal{K}$ of residue characteristic prime to $N_{E(\Pi) / \mathbb{Q}}(\lambda)$,

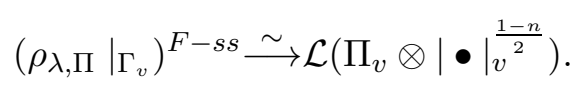


Here the superscript ${ }^{F-s s}$ denotes Frobenius semisimplification.

(b) For all finite primes $v$ of $\mathcal{K}$ dividing $N_{E(\Pi) / \mathbb{Q}}(\lambda),\left.\rho_{\lambda, \Pi}\right|_{\Gamma_{v}}$ is de Rham, and its Hodge-Tate numbers have multiplicity at most one (i.e., $\rho_{\lambda, \Pi}$ is Hodge-Tate regular) and are determined by $\Pi_{\infty}$, or equivalently by $W(\Pi)$, in accordance with the recipe given in (1.5) below.

(c) Let $v$ be a finite prime of $\mathcal{K}$ dividing $N_{E(\Pi) / \mathbb{Q}}(\lambda)$. Suppose $\Pi_{v}$ has a non-zero vector fixed by a maximal compact subgroup of $G L\left(n, \mathcal{K}_{v}\right)$. Then $\rho_{v}:=\left.\rho_{\lambda, \Pi}\right|_{\Gamma_{v}}$ is crystalline, and if $\varphi$ denotes the smallest linear power of the crystalline Frobenius of $D_{\text {crys }}\left(\rho_{v}\right)$ then

$$
\operatorname{det}\left(T-\varphi \mid D_{\text {cris }}\left(\rho_{v}\right)\right)=\operatorname{det}\left(T-\mathcal{L}\left(\left.\Pi_{v} \otimes|\bullet|\right|^{\frac{1-n}{2}}\right)\left(F r o b_{v}\right)\right)
$$

(d) Let $v$ be a finite prime of $\mathcal{K}$ dividing $N_{E(\Pi) / \mathbb{Q}}(\lambda)$. Suppose $\Pi_{v}$ has a non-zero vector fixed by an Iwahori subgroup of $G L\left(n, \mathcal{K}_{v}\right)$. Then $\left.\rho_{\lambda, \Pi}\right|_{\Gamma_{v}}$ is semistable.

(ii) If $n$ is even, the same conclusions hold as in (i), provided $\Pi$ in addition satisfies Special Hypothesis 1.3.

When $\Pi$ satisfies the additional hypothesis that $\Pi_{v_{0}}$ is square-integrable for some finite place $v_{0}$, then this theorem is mostly proved in $[\mathrm{HT}]$, extending an earlier theorem due to Clozel and Kottwitz [C, K2] obtaining (a) at most places where $\Pi$ is unramified. The theorem is completed in [TY]. What we here call $\rho_{\lambda, \Pi}$ is the representation denoted $R_{\ell}\left(\Pi^{\vee}\right)$ in $[\mathrm{HT}]$. The compatibility (a) with the local Langlands correspondence is due in general to Shin [S]. A weaker version of Theorem 1.4 with local compatibility at almost all finite places is deduced in [CHL.IV.3] from the results of [L.IV.1] and [CHL.IV.3], using the methods of [K1].

\subsection{Hodge-Tate numbers of automorphic Galois representations}

Fix a prime $\lambda$ of the coefficient field $E(\Pi)$, say of residue characteristic $p$. The automorphic Galois representation $\rho_{\lambda, \Pi}$ of Theorem 1.4 is obtained in the cohomology of a geometric $p$-adic local system $\tilde{W}_{p}(\Pi)$ on a proper Shimura variety, obtained in a standard way from the finite-dimensional representation $W(\Pi)$ introduced above. It is therefore of geometric type, in the sense of Fontaine and Mazur: each $\rho_{\lambda, \Pi}$ is unramified outside a finite set of places of $\mathcal{K}$, and at every place dividing the residue characteristic of $\lambda$, the representation $\rho_{\lambda, \Pi}$ is de Rham. The latter fact is a consequence of the comparison theorems of $p$-adic Hodge theory. 
The relevant Shimura varieties, say $S h(G)$, are associated to certain unitary similitude groups $G=G U\left(V_{1}\right)$, in the notation of [CHL.IV.2], relative to the $\mathrm{CM}$ extension $\mathcal{K} / F$. They have canonical models as (pro)-varieties over $\mathcal{K}$, and for any open compact $U \subset G\left(\mathbf{A}^{f}\right)$ we have a variety of finite type $S h_{U}(G)$. The group $G$ is quasi-split at each finite place of $F$, and the unitary subgroup $U\left(V_{1}\right)$ is of signature $(1, n-1)$ at the archimedean prime $v_{0}$ of $F$ and is compact at the (non-empty) set of other archimedean places of $F$. Assume that $v$ is a finite place of $F$ which splits in $\mathcal{K}$, and that the compact open subgroup $U_{v}=U \cap U\left(V_{1}\right)\left(\mathbf{A}^{f}\right)$ is maximal (resp. an Iwahori subgroup). Then $S h_{U_{v}}(G)$ has good (resp. semistable) reduction by [Z, LR, $\mathrm{HT}]$ (resp. [TY]). ${ }^{2}$

For the same reasons, $\rho_{\lambda, \Pi}$ is semistable at a prime $v$ dividing the residue characteristic of $\lambda$ such that $\Pi_{v}$ has Iwahori invariants, and when $\Pi_{v}$ is unramified, it is even crystalline. The second part of Thm. 1.4 (c) follows in a standard way from part (a) and theorems of Katz-Messing [KM] and Gillet-Messing [GM]: see [BGGT1, p. 25] for more details.

In particular the Hodge-Tate numbers can be read off from the Hodge numbers of the de Rham cohomology of the flat vector bundle $\tilde{W}(\Pi)$ associated to $W(\Pi)$. The comparison of $\tilde{W}_{p}(\Pi)$ and $\tilde{W}(\Pi)$, and therefore the determination of the Hodge-Tate numbers from the highest weights $\mu(\sigma)$ of $W_{\sigma}$, presupposes a dictionary relating complex and $p$-adic places of $\mathcal{K}$. In $[\mathrm{HT}]$ this is given by an isomorphism $\iota: \overline{\mathbb{Q}}_{p} \stackrel{\sim}{\longrightarrow} \mathbb{C}$. For what follows it suffices to identify the algebraic closure of $\mathbb{Q}$ in $\overline{\mathbb{Q}}_{p}$ with the field of algebraic numbers in $\mathbb{C}$. Then the $p$-adic embeddings of $\overline{\mathbb{Q}}$, and in particular of $\mathcal{K}$, are identified with the complex embeddings; if $s$ is an embedding of $\mathcal{K}$ in $\overline{\mathbb{Q}}_{p}$, we write $\iota(s)$ for the corresponding complex embedding. Let $\overline{s(\mathcal{K})}$ denote the closure of $s(\mathcal{K})$ in $\overline{\mathbb{Q}}_{p}$.

Let $s$ be an embedding of $\mathcal{K}$ in $\overline{\mathbb{Q}}_{p}$, and let $D_{d R, s}$ denote Fontaine's functor from $E(\Pi)_{\lambda}$-representations of $\Gamma_{s}=\operatorname{Gal}\left(\overline{\mathbb{Q}}_{p} / \overline{s(\mathcal{K})}\right)$ to filtered $E(\Pi)_{\lambda} \otimes_{\mathbb{Q}_{p}}$

\footnotetext{
${ }^{2}$ If $v$ is unramified over $\mathbb{Q}$, the statement that the relevant Shimura variety at finite level has good reduction at $v$ is in [K3]; Kottwitz attributes the result to Zink [Z] and Langlands-Rapoport [LR]. There's no question that the method of proof of Satz 6.2 of [LR] suffices for the proof, but the authors refer to the proof as a "Skizze"; Zink states his theorem under local hypotheses that are superfluous for the proof of good reduction. When $v$ is not assumed unramified over $\mathbb{Q}$, the proof of good reduction is in $[\mathrm{HT}]$, again under a superfluous local hypothesis. Similarly, [TY] makes the assumption that $G$ is the unitary similitude group attached to a division algebra over $\mathcal{K}$, which is again unnecessary to prove semistable reduction. Zink also proves that the reduction is proper, again under unnecessarily restrictive hypotheses.
} 
$\overline{s(\mathcal{K})}$-modules:

$$
D_{d R, s}(R)=\left(R \otimes_{\mathbb{Q}_{p}} B_{d R}\right)^{\Gamma_{s}} .
$$

The Hodge-Tate numbers of $R$ (with respect to $s$ ) are the $j$ such that $g r^{j} D_{d R, s}(R) \neq(0)$; the corresponding graded pieces are free of rank 1 over $E(\Pi)_{\lambda} \otimes_{\mathbb{Q}_{p}} \overline{s(\mathcal{K})}$. Then in the situation of Theorem 1.4, the Hodge-Tate numbers of $\rho_{\lambda, \Pi}$ with respect to $s$ are the $j$ of the form

$$
j=\mu_{i}(\iota(s))+n-i, \quad i=1, \ldots, n .
$$

This is to be compared to part 4 of Theorem VII.1.9 of [HT]; the replacement of $\iota(s)$ by $\iota(s)^{c}$ corresponds to our replacement of $\Pi$ by $\Pi^{\vee}$ in the definition of $\rho_{\lambda, \Pi \cdot{ }^{3}}$

Suppose $n$ is even but $\Pi$ does not satisfy Hypothesis 1.3. Part (a) of the following theorem is proved (but not stated) by Clozel in [C3] and is stated as well as proved as part of a much more general theorem by Scholze and Shin $[\mathrm{SS}]$ :

Theorem 1.7. Suppose $\Pi$ satisfies Hypotheses (1.1) and (1.2). Then there is a number field $E(\Pi)$ and a compatible system $\rho_{\lambda, \Pi}: \Gamma_{\mathcal{K}} \rightarrow G L\left(\frac{n(n-1)}{2}, E(\Pi)_{\lambda}\right)$ of semi-simple $\lambda$-adic representations, where $\lambda$ runs through the finite places of $E(\Pi)$, such that

(a) For almost all finite primes $v$ of $\mathcal{K}$ of residue characteristic prime to $N_{E(\Pi) / \mathbb{Q}}(\lambda)$ at which $\Pi_{v}$ is unramified, $\rho_{\lambda, \Pi}$ is an unramified representation, and

$$
\left.\rho_{\lambda, \Pi}\right|_{\Gamma_{v}} ^{s s} \stackrel{\sim}{\longrightarrow} \wedge^{2} \mathcal{L}\left(\Pi_{v}\right)(1-n) .
$$

(b) For all finite primes $v$ of $\mathcal{K}$ dividing $N_{E(\Pi) / \mathbb{Q}}(\lambda),\left.\rho_{\lambda, \Pi}\right|_{\Gamma_{v}}$ is de Rham.

(c) Let $v$ be a finite prime of $\mathcal{K}$, unramified over $\mathbb{Q}$ and dividing $N_{E(\Pi) / \mathbb{Q}}(\lambda)$. Suppose $\Pi_{v}$ has a non-zero vector fixed by a maximal compact subgroup of $G L\left(n, \mathcal{K}_{v}\right)$. Then $\left.\rho_{\lambda, \Pi}\right|_{\Gamma_{v}}$ is crystalline.

This theorem is equally valid for odd and even $n$, but is a consequence of Theorem 1.4 when $n$ is odd.

Proof. Theorem 1.7 is the Galois counterpart of one of the theorems about stable base change for unitary groups proved in [L.IV.1]. In [C3] the relevant reference is the equality between the expressions (3.7) and (3.8); in [SS]

\footnotetext{
${ }^{3}$ In comparing the formulas here with those in $[\mathrm{HT}]$, the reader is warned that highest weights of representations of $G L(n)$ are indexed in [HT] by increasing $n$ tuples.
} 
part (a) of the theorem is a special case of Theorem 10.2. Part (b) follows from the fundamental comparison theorem between $p$-adic étale cohomology and de Rham cohomology (Fontaine's $C_{D R}$, now proved by many people). Part (c) follows similarly from Fontaine's $C_{c r i s}$ and the fact that, under the hypotheses, the representation $\rho_{\lambda, \Pi}$ is realized in the cohomology of a Shimura variety with good reduction at $v[\mathrm{Z}, \mathrm{LR}]{ }^{4}$

\section{An application of eigenvarieties and $p$-adic families of Galois representations}

In this section we recall the main result of [Ch] (using Theorem 1.4 above).

Let $\mathcal{K} / F$ be a $C M$ quadratic extension of a totally real field, satisfying Special Hypotheses (1.2.1) and (1.2.3), and assume $n$ even. These hypotheses imply by a standard Galois cohomological argument (cf. [C2]) that

Lemma 2.1. There exists a hermitian space $V_{0} / \mathcal{K}$ relative to the extension $\mathcal{K} / F$ such that the unitary group $G_{0}=U\left(V_{0}\right)$ satisfies

(i) For all finite places $v, G_{0}\left(F_{v}\right)$ is quasi-split and splits over an unramified extension of $F_{v}$; in particular, $G_{0}\left(F_{v}\right)$ contains a hyperspecial maximal compact subgroup.

(ii) For all real places $v, G_{0}\left(F_{v}\right)$ is compact.

Moreover, $G_{0}$ is unique up to isomorphism.

By Labesse [L.IV.1], any ПI satisfying Hypotheses (1.1) and (1.2) admits a strong descent to the unitary group $G_{0}$.

Fix $\ell$ a finite prime and consider the following hypothesis on a $\Pi$ satisfying Hypotheses (1.1).

Special Hypotheses 2.2. There is a place $v_{0}$ of $F$ dividing the rational prime $\ell$ such that

(2.2.1) $v_{0}$ splits in $\mathcal{K}$,

(2.2.2) If $v$ is a place of $\mathcal{K}$ dividing $v_{0}, \Pi_{v}$ has nonzero Iwahori-invariants.

We fix a pair of embeddings $\iota=\left(\iota_{\ell}, \iota_{\infty}\right)$ of $\overline{\mathbb{Q}}$ into $\overline{\mathbb{Q}}_{\ell}$ and $\mathbb{C}$. The following theorem is [Ch, Thm. $3.3 \& 3.5]$. If $\rho=(s, N)$ and $\rho^{\prime}=\left(s^{\prime}, N^{\prime}\right)$ are two Weil-Deligne representations, we refer to $[\mathrm{Ch}] \S 3.1$ for the precise

\footnotetext{
${ }^{4} \mathrm{As}$ in note $1,[\mathrm{Z}]$ proves good reduction under somewhat restrictive hypotheses at a prime unrelated to $v$, but the proof works without these hypotheses. The assumption that $v$ be unramified over $\mathbb{Q}$ is certainly unnecessary but the more general case has not been treated in the literature.
} 
definition of the dominance relation $\rho \prec \rho^{\prime}$. Let us simply say here that it implies that $s \simeq s^{\prime}$ and that $N$ is in the Zariski-closure of the conjugacy class of $N^{\prime}$.

Theorem 2.3. Suppose $\Pi$ satisfies Hypotheses (1.1), (1.2) and Special Hypotheses (2.2) (but not necessarily (1.3)). Then there is a semisimple continuous Galois representation $\rho_{\iota, \Pi}: \Gamma_{\mathcal{K}} \rightarrow G L\left(n, \overline{\mathbb{Q}}_{\ell}\right)$ such that

$\left(\mathrm{a}^{\prime}\right)$ For all finite primes $v$ of $\mathcal{K}$ of residue characteristic prime to $\ell$,

$$
\left.\rho_{\iota, \Pi}^{F-s s}\right|_{\Gamma_{v}} \prec \mathcal{L}\left(\Pi_{v} \otimes|\bullet|_{v^{\frac{1-n}{2}}}\right) .
$$

Moreover, the assertion about Hodge-Tate numbers in Theorem 1.4. (i) (b) holds, as well as the whole of (b), (c) and (d) for all finite primes $v$ of $\mathcal{K}$ above $\ell$ but not dividing $v_{0}$.

The main ingredient in the proof is the construction of certain $\ell$-adic eigenvarieties of the unitary group $G_{0}$. These eigenvarieties are chosen so as to interpolate $\ell$-adically all the automorphic representations $\pi$ of $G_{0}$ which are unramified at the finite places $v$ of $F$ that are inert in $\mathcal{K}$ and such that:

- $\pi_{v} \simeq W_{v}^{\vee}$ for each archimedean prime $v$ of $F$ such that the $\ell$-adic place of $F$ defined by $\iota_{\ell} \iota_{\infty}^{-1}(v)$ is not the given place $v_{0}$; here we identify irreducible representations of the compact Lie group $U(n)$ with irreducible algebraic representations of $G L(n)$.

- for each finite prime $v$ of $F$ that splits in $\mathcal{K}, \pi_{v}$ and $\Pi_{v}$ are in the same Bernstein component and the monodromy operator of $\mathcal{L}\left(\pi_{v}\right)$ is dominated by the one of $\mathcal{L}\left(\Pi_{v}\right)$ (see [BC, §6.5] for a more precise condition).

The inertial part in $\left(\mathrm{a}^{\prime}\right)$, including the assertion about the monodromy operator, is due to Bellaïche-Chenevier [BC]. Moreover, parts (b), (c) and (d) rely on the work of Berger and Colmez on families of $\ell$-adic Galois representations of $\ell$-adic fields with constant Hodge-Tate weights [BCo].

\section{Removal of Special Hypotheses}

\subsection{Removal of Special Hypotheses 1.2}

Let $\mathcal{I}$ denote a set of cyclic extensions of a fixed number field $E$, each being of the same prime degree $d$, and let $S$ be a finite set of places of $E$. The set $\mathcal{I}$ is called $S$ general [So, Definitions 1 and 3] if, for any finite extension $M$ 
of $E$, and any prime $v$ of $E$ not in $S$, there is $E_{i} \in \mathcal{I}$ linearly disjoint from $M$ such that $v$ splits completely in $E_{i}$. This notion is useful for patching Galois representations. Indeed, fix some integer $n \geq 1$, a prime $\ell$, as well as a semisimple continuous representation

$$
\rho_{i}: \Gamma_{E_{i}} \rightarrow G L\left(n, \overline{\mathbb{Q}}_{\ell}\right)
$$

for each $E_{i} \in \mathcal{I}$. Assume that for all $i, j$ we have $\rho_{i \mid \Gamma_{E_{i} E_{j}}} \simeq \rho_{j \mid \Gamma_{E_{i} E_{j}}}$ and $\rho_{i}^{\sigma_{i}} \simeq \rho_{i}$, where $\sigma_{i}$ is a generator of $\operatorname{Gal}\left(E_{i} / E\right)$. Then the main result of [So], extending an argument of Blasius-Rogawski when $d=2$ and of the second author in general, ensures that there exists a unique semisimple continuous representation $\rho: \Gamma_{E} \rightarrow G L\left(n, \overline{\mathbb{Q}}_{\ell}\right)$ such that $\rho_{\mid \Gamma_{E_{i}}} \simeq \rho_{i}$ for each $i$.

Let now $\mathcal{K} / F$ be any $\mathrm{CM}$ quadratic extension of a totally real field, and let $\Pi$ be a cuspidal automorphic representation of $G L(n, \mathcal{K})$ satisfying Hypothesis 1.1 .

Proposition 3.1.1. There is a finite set $S$ of places of $F$ and an $S$-general collection $\mathcal{I}$ of totally real quadratic extensions $F_{i} / F$ such that, for each $F_{i} \in \mathcal{I}$, letting $\mathcal{K}_{i}=F_{i} \cdot \mathcal{K}, \Pi_{i}$ the base change of $\Pi$ to $\mathcal{K}_{i}$, the triple $\left(F_{i}, \mathcal{K}_{i}, \Pi_{i}\right)$ satisfies Special Hypotheses 1.2. Moreover, we can assume that, for every $v \in S$ and every $F_{i} \in \mathcal{I}$, either $v$ splits in $\mathcal{K} / F$ or the unique extension of $v$ to $\mathcal{K}$, denoted $\tilde{v}$, splits in $\mathcal{K}_{i}$.

Proof. Let $S$ be the set of primes $v$ of $F$ at which (1.2.1) or (1.2.2) fails: either $v$ ramifies in $\mathcal{K} / F$, or $v$ stays prime in $\mathcal{K}$ and the corresponding component $\Pi_{\tilde{v}}$ is ramified. We take $\mathcal{I}$ to be the set of totally real quadratic extensions $F_{i} / F$ with the property that, for all $v \in S, F_{i, v} \stackrel{\sim}{\longrightarrow} \mathcal{K}_{\tilde{v}}$ where $F_{i, v}=F_{i} \otimes_{F} F_{v}$. We also assume that each $F_{i} / F$ is ramified at at least one finite place not in $S$ at which $\Pi$ is unramified. This last condition ensures that $\Pi_{i}$ is cuspidal. It is obvious that this set has the properties claimed.

Theorem 3.1.2. Let $\Pi$ be a cuspidal automorphic representation of $G L(n, \mathcal{K})$ satisfying Hypothesis 1.1 and Special Hypothesis 1.3 if $n$ is even. Then the conclusions of Theorem 1.4 also hold for $\Pi$.

If $n$ is even and satisfies Hypothesis 1.1, then the conclusions of Theorem 1.7 also hold for $\Pi$.

Proof. Observe that the collection $\left\{\mathcal{K}_{i}\right\}$ of quadratic extensions of $\mathcal{K}$ given by Proposition 3.1.1 is $S^{\prime}$-general, where $S^{\prime}$ is the set of places of $\mathcal{K}$ above $S$. The first part follows from Theorem 1.4 and the patching lemma of [So] recalled above (see [HT] pp. 229-232 for more details). The second assertion is deduced from Theorem 1.7 and [So] in the same way. 


\subsection{Removal of Special Hypotheses 1.3}

Since Special Hypotheses 1.3 are only relevant to even $n$, we assume $n$ to be even. Moreover, the case $n=2$ is already understood. Thus we assume $n \geq 4$.

Lemma 3.2.1. Let $F$ be a totally real field, $v$ a finite place of $F, S$ a finite set of places of $F$ not containing $v, M / F$ any finite extension. Let $L / F_{v}$ be a cyclic extension of prime degree $d$. There exists a totally real cyclic extension $F^{\prime} / F$ of degree $d$ in which any place of $S$ splits completely, linearly disjoint from $M$, such that, for the unique place $v^{\prime}$ of $F^{\prime}$ dividing $v$, the extension $F_{v^{\prime}}^{\prime} / F_{v}$ is isomorphic to $L / F_{v}$.

Proof. Fix $M$ as in the statement. By enlarging $S$ if necessary, we may assume that $S$ contains the infinite places of $F$ and that for any cyclic subextension $C / F$ of $M / F$, there is a place in $S$ which is not split in $C$ (Chebotarev). Any cyclic extension of $F$ in which every place of $S$ splits completely is linearly disjoint from $M$ (this argument is taken from [CHT] Lemma 4.1.2). The lemma follows then from the Grunwald-Wang theorem $[\mathrm{AT}]$. Note that we are not in the so-called "special case" as $d$ is prime.

Fix some rational prime $\ell$. Define the solvable index of a finite Galois extension of $\ell$-adic fields $L / K$ as the integer $m \geq 0$ such that there is a tower of extensions

$$
L=L_{m} \supset L_{m-1} \supset \cdots \supset L_{0}=K
$$

with each $L_{i+1} / L_{i}$ cyclic of prime degree. For an integer $m \geq 0$, consider the following property of a cuspidal automorphic representation $\Pi$ of $\mathcal{G}$ satisfying (1.1).

Property $\mathbf{P}(\mathbf{m})$. There is a prime $v$ of $F$ dividing $\ell$ and that splits as $v=u u^{\prime}$ in $\mathcal{K}$, as well as a Galois extension $L / F_{v}$ of solvable index $m$, such that the base change of $\Pi_{u}$ to $L$ has a nonzero Iwahori fixed vector.

If $\Pi$ satisfies $\mathrm{P}(\mathrm{m})$ and if $v$ is as above we will say that $\Pi$ satisfies $\mathrm{P}(\mathrm{m})$ at $v$. It follows from the local Langlands correspondence, and indeed from the numerical correspondence proved by Henniart, that for any $\Pi$, and any place $v=u u^{\prime}$ of $F$ dividing $\ell$ that splits in $\mathcal{K}$, there exists an integer $m$ such that $\Pi$ satisfies $\mathrm{P}(\mathrm{m})$ at $v$. More precisely, letting $(s, N)$ be the representation of the Weil-Deligne group of $F_{v}$ corresponding to $\Pi_{u}-s$ is a Frobenius semisimple representation of the Weil group of $F_{v}$ and $N$ is a nilpotent endomorphism satisfying the usual commutation rules - we choose $L$ such that the restriction of $s$ to the Weil group of $L$ is unramified. 
Corollary 3.2.2. Let $m$ be an integer and let $\Pi$ be a cuspidal automorphic representation of $\mathcal{G}$ satisfying Hypotheses 1.1 and property $P(m+1)$ at the place $v$. Let $d$ be the prime degree of a cyclic extension $L_{1} / F_{v}$ given by the property $P(m+1)$. Let $M / F$ be any finite extension. Let $w$ be a place of $F$ different from $v$.

There is a totally real cyclic extension $F^{\prime} / F$ of degree $d$ in which $w$ splits completely, linearly disjoint from $M$, and with a unique place $v^{\prime}$ above $v$, such that, letting $\mathcal{K}^{\prime}=\mathcal{K} \cdot F^{\prime}$, the base change $\Pi_{\mathcal{K}^{\prime}}$ of $\Pi$ to $G L\left(n, \mathcal{K}^{\prime}\right)$ is cuspidal and satisfies $P(m)$ at the place of $F^{\prime}$ dividing $v$.

Proof. By enlarging $M$ if necessary, we may assume that $M$ contains any cyclic extension $E / F$ such that the base-change of $\Pi$ to $G L(n, E \cdot \mathcal{K})$ is not cuspidal. That there are only finitely many such extensions follows from Arthur-Clozel's base change. Apply the previous lemma to $F, v, S=\{w\}$, $M$ and $L=L_{1}$.

Remark. It follows that the collection $\mathcal{I}$ of totally real cyclic extensions $F^{\prime} / F$ of degree $d$ for which $\Pi_{\mathcal{K}^{\prime}}$ satisfies $\mathrm{P}(\mathrm{m})$ at $v$ is $\{v\}$-general. The collection of cyclic extensions $\mathcal{K} F^{\prime} / \mathcal{K}$ with $F^{\prime} \in \mathcal{I}$ is thus $\left\{u, u^{\prime}\right\}$-general as well, where $v=u u^{\prime}$ in $\mathcal{K}$.

Theorem 3.2.3. Fix a prime $\ell$ and embeddings $\iota=\left(\iota_{\ell}, \iota_{\infty}\right)$ as above. Let $\Pi$ be a cuspidal automorphic representation of $G L(n, \mathcal{K})$ satisfying General Hypotheses (1.1). Then there exists a semisimple continuous Galois representation

$$
\rho_{\iota, \Pi}: \Gamma_{\mathcal{K}} \rightarrow G L\left(n, \overline{\mathbb{Q}}_{\ell}\right)
$$

satisfying the following conditions:

$\left(\mathrm{a}^{\prime}\right)$ For all finite primes $v$ of $\mathcal{K}$ of residue characteristic prime to $\ell$,

$$
\left(\left.\rho_{\iota, \Pi}\right|_{\Gamma_{v}}\right)^{F-s s} \prec \mathcal{L}\left(\Pi_{v} \otimes|\bullet|_{v^{\frac{1-n}{2}}}\right),
$$

where $\Gamma_{v}$ is a decomposition group at $v, \mathcal{L}$ is the local Langlands correspondence, and the relation $\prec$ is the partial ordering on the associated Weil-Deligne representations defined in [Ch] §3.1;

(b) For all finite primes $v$ of $\mathcal{K}$ dividing $\ell,\left.\rho_{\lambda, \Pi}\right|_{\Gamma_{v}}$ is de Rham, and its Hodge-Tate numbers have multiplicity at most one (i.e., $\rho_{\lambda, \Pi}$ is HodgeTate regular) and are determined by $\Pi_{\infty}$, or equivalently by $W(\Pi)$, in accordance with the recipe given in (1.5).

(c) Let $v$ be a finite prime of $\mathcal{K}$ dividing $\ell$. Suppose $\Pi_{v}$ has a non-zero vector fixed by a maximal compact subgroup of $G L\left(n, \mathcal{K}_{v}\right)$. Then $\rho_{v}:=$ 
$\left.\rho_{\lambda, \Pi}\right|_{\Gamma_{v}}$ is crystalline, and if $\varphi$ denotes the smallest linear power of the crystalline Frobenius of $D_{\text {crys }}\left(\rho_{v}\right)$ then

$$
\operatorname{det}\left(T-\varphi \mid D_{\text {cris }}\left(\rho_{v}\right)\right)=\operatorname{det}\left(T-\mathcal{L}\left(\Pi_{v} \otimes|\bullet| v^{\frac{1-n}{2}}\right)\left(F_{r o b}\right)\right) .
$$

(d) Let $v$ be a finite prime of $\mathcal{K}$ dividing $\ell$. Suppose $\Pi_{v}$ has a non-zero vector fixed by an Iwahori subgroup of $G L\left(n, \mathcal{K}_{v}\right)$. Then $\left.\rho_{\lambda, \Pi}\right|_{\Gamma_{v}}$ is semistable.

Proof. Arguing as in Theorem 3.1.2 we may assume that $\mathcal{K} / F$ satisfies Hypothesis (1.2) and that furthermore each prime of $F$ dividing $\ell$ splits in $\mathcal{K}$. If $F^{\prime} / F$ is a totally real field, note that $\mathcal{K} F^{\prime} / F^{\prime}$ still has these properties. The discussion following the statement of property $\mathrm{P}(\mathrm{m})$ ensures moreover that for any place $v$ dividing $\ell$ there is some $m \geq 0$ such that $\Pi$ satisfies $\mathrm{P}(\mathrm{m})$ at $v$.

We first show by induction on the integer $m \geq 0$ that for any $(F, \mathcal{K}, \Pi)$ with $\mathcal{K} / F$ as above and satisfying $\mathrm{P}(\mathrm{m})$ at some place $v$ above $\ell$, there exists a (necessarily) unique $\rho_{\iota, \Pi}$ satisfying $\left(\mathrm{a}^{\prime}\right)$, which moreover satisfies (b), (c) and (d) at each place $v^{\prime}$ dividing $\ell$ but not dividing $v$. When $m=0$ this is exactly Theorem 2.3. Assume now by induction that this property holds for the integer $m \geq 0$ and assume that $\Pi$ satisfies $\mathrm{P}(\mathrm{m}+1)$ at the place $v$ of $F$. By the previous Remark and Chebotarev, we may patch the $\rho_{\iota, \Pi_{F^{\prime}} \mathcal{K}}$ along the $\left\{u, u^{\prime}\right\}$-general collection $\left\{F^{\prime} \mathcal{K}, F^{\prime} \in \mathcal{I}\right\}$, where $v=u u^{\prime}$ in $\mathcal{K}$. Define $\rho_{\iota, \Pi}$ as the resulting representation of $\Gamma_{\mathcal{K}}$. It is immediate that it has the required properties, which concludes the induction.

The recipe for the Hodge-Tate numbers at the primes dividing $\ell$ follows from this construction, as they can be read off after a finite base change, and the relevant assertion in Theorem 2.3. In particular, $\rho_{\iota, \Pi}$ is Hodge-Tate at any prime dividing $\ell$. Better, we have shown that given an $\ell$-adic place $v$ of $F$ then (b), (c) and (d) hold at each $\ell$-adic place of $\mathcal{K}$ not dividing $v$. In particular, we are done if $F$ has at least two $\ell$-adic places. But we can always reduce to this case by a suitable totally real quadratic base change $F^{\prime} / F$ in which each $\ell$-adic place of $F$ splits. This concludes the proof.

We now offer a second argument proving that $\rho_{\iota, \Pi}$ is de Rham at each place dividing $\ell$ which uses Theorem 1.7 (which has not been used so far) but less material from the theory of $\ell$-adic families of Galois representations of $\ell$-adic fields. More precisely, we do not assume the last assertion in Theorem 2.3 concerning (b), (c) and (d) (based on the aforementioned work of Berger and Colmez), but only the one concerning the Hodge-Tate numbers (a consequence of Sen's theory for families of Galois representations). 
Part (b) of Theorem 1.7, together with condition (a) and Chebotarev density, implies at least that $\wedge^{2} \rho_{\iota, \Pi}$ is de Rham. Now since $n \geq 4$, the map from $\wedge^{2}: G L(n) \rightarrow G L\left(\frac{n(n-1)}{2}\right)$ is an isogeny. A theorem of Wintenberger [Wi] asserts that if $L$ is an $\ell$-adic field and $\rho: \Gamma_{L} \rightarrow G L\left(n, \overline{\mathbb{Q}}_{\ell}\right)$ is a Hodge-Tate representation whose image under an isogeny is de Rham, then $\rho$ is itself de Rham. When $\ell$ is unramified in $F$, a similar argument shows that $\rho$ is crystalline at $v$ when $\Pi_{v}$ has a non-zero vector fixed by a maximal compact subgroup of $G L\left(n, \mathcal{K}_{v}\right)$.

Remark 3.2.4. As far as we know, the idea of the proof given here to get properties (b), (c) and (d), which relies on the last assertion in Theorem 2.3 (hence on the use of an $\ell$-adic eigenvariety with only partially moving weights to apply the results of Berger-Colmez) is new. Christopher Skinner informed us that he also had this idea to get part (c) of the result above.

The following result (and its proof) is due to $\mathrm{R}$. Taylor. If $E \subset \mathbb{C}$ is a number field, and if $\iota$ is a pair of embeddings as above, we denote by $E_{\iota}$ be the closure of $\iota_{\ell} \iota_{\infty}^{-1}(E)$ inside $\overline{\mathbb{Q}}_{\ell}$.

Proposition 3.2.5. Let $\Pi$ be a cuspidal automorphic representation of $\mathcal{G}$ satisfying Hypotheses 1.1. There is a number field $E(\Pi) \subset \mathbb{C}$ such that for each pair of embeddings $\iota$, the representation $\rho_{\iota, \Pi}$ is defined over $E(\Pi)_{\iota}$.

The authors do not know however if it is always the case that each $\rho_{\iota, \Pi}$ is defined over the finite extension of $\mathbb{Q}_{\ell}$ generated by its traces.

Proof. Let $E_{0}(\Pi)$ be the coefficient field of the cohomological representation $\Pi\left[\mathrm{C} 1\right.$, Proposition 3.1]. By property $\left(\mathrm{a}^{\prime}\right)$, the characteristic polynomial $\chi_{v}$ of the image under $\rho_{\iota, \Pi}$ of a Frobenius at a finite place $v$ of $\mathcal{K}$ belongs to $E_{0}(\Pi)[T]$ and is independent of $\iota$ (assuming that $\Pi_{v}$ is unramified and that $\ell$ is chosen prime to $v$ ). By Chebotarev's theorem the field of traces of $\rho_{\iota, \Pi}$ is thus included in $E_{0}(\Pi)_{\iota}$.

Let $G$ be a group, $L / K$ a field extension, and $\rho: G \rightarrow G L_{n}(L)$ a semisimple representation whose traces belong to $K$. Recall that if there is an element $g \in G$ such that the characteristic polynomial of $\rho(g)$ has $n$ distinct roots in $K$, then $\rho$ is defined over $K$. (A central simple algebra over $K$ of dimension $n^{2}$ with $n$ mutually orthogonal idempotents is isomorphic to the matrix algebra.) It is thus enough to show that there exist two finite places $v_{1}, v_{2}$ of $\mathcal{K}$ of distinct residue characteristic such that $\chi_{v_{1}}$ and $\chi_{v_{2}}$ each have $n$ distinct roots in $\mathbb{C}$ : any number field $E(\Pi)$ containing $E(\Pi)_{0}$ and those roots does the trick.

Fix some prime $\ell$ and a pair $\iota$. Let $H$ denote the Zariski closure of $\rho_{\iota, \Pi}\left(\Gamma_{\mathcal{K}}\right)$ in $G L_{n}\left(\overline{\mathbb{Q}}_{\ell}\right)$. As $\rho_{\iota, \Pi}$ is Hodge-Tate with distinct Hodge-Tate weights 
for any embedding $\mathcal{K} \rightarrow \overline{\mathbb{Q}}_{\ell}$ (one would be enough), it follows from an argument of Serre that the $\overline{\mathbb{Q}}_{\ell}$-group $H$ contains an element with $n$ distinct eigenvalues [Se, §3]. We conclude the proof by Chebotarev's theorem.

\section{Totally real fields}

We now state a version of Theorem 1.4 for cohomological automorphic representations of $G L\left(n, \mathbf{A}_{F}\right)$. General Hypotheses 1.1 are replaced by the following hypothesis.

General Hypotheses 4.1. Let $\Pi$ be a cuspidal automorphic representation of $G L\left(n, \mathbf{A}_{F}\right)$. Writing $\Pi=\Pi_{\infty} \otimes \Pi_{f}$, we assume

(i) (Regularity) There is a finite-dimensional irreducible representation $W(\Pi)$ of $\mathcal{G}(\mathbb{R})$ such that

$$
H^{*}\left(\mathfrak{g}, K_{\infty} ; \Pi_{\infty} \otimes W(\Pi)\right) \neq 0
$$

(ii) (Polarization) There is a Hecke character

$$
\chi: \mathbf{A}_{F}^{\times} / F^{\times} \rightarrow \mathbb{C}^{\times}
$$

with $\chi_{v}(-1)$ independent of the prime $v \mid \infty$ such that the contragredient $\Pi^{\vee}$ of $\Pi$ satisfies

$$
\Pi^{\vee} \stackrel{\sim}{\longrightarrow} \Pi \otimes \chi
$$

Theorem 4.2. Let $\Pi$ be a cuspidal automorphic representation of $G L\left(n, \mathbf{A}_{F}\right)$ satisfying Hypotheses 4.1. Then there is a number field $E(\Pi)$ and a compatible system $\rho_{\lambda, \Pi}: \Gamma_{F} \rightarrow G L\left(n, E(\Pi)_{\lambda}\right)$ of $\lambda$-adic representations, where $\lambda$ runs through the finite places of $E(\Pi)$, such that

$\left(\mathrm{a}^{\prime}\right)$ For all finite primes $v$ of $F$ of residue characteristic prime to $N_{E(\Pi) / \mathbb{Q}}(\lambda)$,

$$
\left(\left.\rho_{\iota, \Pi}\right|_{\Gamma_{v}}\right)^{F-s s} \prec \mathcal{L}\left(\Pi_{v} \otimes|\bullet|_{v^{2}}^{\frac{1-n}{2}}\right) .
$$

Here $\Gamma_{v}$ denotes a decomposition group at $v$.

(b) For all finite primes $v$ of $F$ dividing $N_{E(\Pi) / \mathbb{Q}}(\lambda),\left.\rho_{\lambda, \Pi}\right|_{\Gamma_{v}}$ is de Rham, and its Hodge-Tate numbers have multiplicity at most one (i.e., $\rho_{\lambda, \Pi}$ is Hodge-Tate regular) and are determined by $\Pi_{\infty}$, or equivalently by $W(\Pi)$, in accordance with the recipe given in (1.5). 
(c) Let $v$ be a finite prime of $F$ dividing $N_{E(\Pi) / \mathbb{Q}}(\lambda)$. Suppose $\Pi_{v}$ has a non-zero vector fixed by a maximal compact subgroup of $G L\left(n, \mathcal{K}_{v}\right)$. Then $\rho_{v}:=\left.\rho_{\lambda, \Pi}\right|_{\Gamma_{v}}$ is crystalline, and if $\varphi$ denotes the smallest linear power of the crystalline Frobenius of $D_{\text {crys }}\left(\rho_{v}\right)$ then

$$
\operatorname{det}(T-\varphi)=\operatorname{det}\left(T-\mathcal{L}\left(\Pi_{v} \otimes|\bullet| \frac{1-n}{v^{2}}\right)\left(F r o b_{v}\right)\right)
$$

(d) Let $v$ be a finite prime of $\mathcal{K}$ dividing $N_{E(\Pi) / \mathbb{Q}}(\lambda)$. Suppose $\Pi_{v}$ has a non-zero vector fixed by an Iwahori subgroup of $G L\left(n, \mathcal{K}_{v}\right)$. Then $\left.\rho_{\lambda, \Pi}\right|_{\Gamma_{v}}$ is semistable.

Proof. The deduction of this result from Theorem 3.2.5 follows exactly the proof of Proposition 4.3.1 of [CHT]. The claim regarding $E(\Pi)$ follows as in Proposition 3.2.5.

Remark 4.3. As in Theorem 2.3, the present methods only allow us to prove $\left(\mathrm{a}^{\prime}\right)$ in general and not the analogue of (a) as in Theorem 1.4. This has since been done by Caraiani [Ca].

\section{References}

[AT] E. Artin and J. Tate, Class field theory, W. A. Benjamin (1968).

[BGGT1] T. Barnet-Lamb, T. Gee, D. Geraghty, and R. Taylor, Potential automorphy and change of weight, preprint at http:// www.math.harvard.edu.

[BGGT2] T. Barnet-Lamb, T. Gee, D. Geraghty, and R. Taylor, Localglobal compatibility for $\ell=p$, II, Annales Scientifique de l'ENS, to appear.

[BGHT] T. Barnet-Lamb, D. Geraghty, M. Harris, and R. Taylor, A family of Calabi-Yau varieties and potential automorphy, II, Publ. RIMS, 47 (2011), 29-98.

[BC] J. Bellaïche and G. Chenevier, Families of Galois representations and Selmer groups, Astérisque 324 (2009).

[BRa] D. Blasius and D. Ramakrishnan, Maass forms and Galois representations, in Galois groups over $\mathbb{Q}$ (Berkeley, CA, 1987), Math. Sci. Res. Inst. Publ., 16, Springer, New York (1989), 33-77.

[BR1] D. Blasius and J. Rogawski, Tate classes and arithmetic quotients of the two-ball, in The zeta functions of Picard modular surfaces, Univ. Montréal, Montréal (1992), 421-444. 
[BR2] D. Blasius and J. Rogawski, Motives for Hilbert modular forms, Inventiones Math., 114 (1993), 55-87.

[Ca] A. Caraiani, Local-global compatibility and the action of monodromy on nearby cycles, Duke Mathematical Journal, 161 (2012), 2311-2413.

[Ch] G. Chenevier, Une application des variétés de Hecke des groupes unitaires, Stabilization of the trace formula, Shimura varieties, and arithmetic applications. Volume 2, in preparation.

[C1] L. Clozel, Motifs et formes automorphes: applications du principe de fonctorialité, In L. Clozel and J. S. Milne, eds., Automorphic forms, Shimura varieties, and L-functions, Vol. I, Perspect. Math., 10 (1990), 77-159.

[C2] L. Clozel, Représentations Galoisiennes associées aux représentations automorphes autoduales de GL(n), Publ. Math. I.H.E.S., 73, 97-145 (1991).

[C3] L. Clozel, Purity reigns supreme, Int. Math. Res. Notices (2012), online January 12, 2012.

[CHL.IV.2] L. Clozel, M. Harris, and J.-P. Labesse, Endoscopic transfer, in L. Clozel, M. Harris, J.-P. Labesse, and B. C. Ngô, eds., Stabilization of the trace formula, Shimura varieties, and arithmetic applications. Volume 1: On the stabilization of the trace formula, Boston: International Press (2011), 475-496.

[CHL.IV.3] L. Clozel, M. Harris, and J.-P. Labesse, Construction of automorphic Galois representations, I, in L. Clozel, M. Harris, J.P. Labesse, and B. C. Ngô, eds., Stabilization of the trace formula, Shimura varieties, and arithmetic applications. Volume 1: On the stabilization of the trace formula, Boston: International Press (2011), 497-527.

[CHT] L. Clozel, M. Harris, and R. Taylor, Automorphy for some $\ell$-adic lifts of automorphic mod $\ell$ Galois representations, Publ. Math. IHES, 1-181.

[GM] H. Gillet and W. Messing, Cycle classes and Riemann-Roch for crystalline cohomology, Duke Math. J., 55 (1987), 501-538.

[HT] M. Harris and R. Taylor, The geometry and cohomology of some simple Shimura varieties, Annals of Mathematics Studies, 151 (2001). 
[K1] R. Kottwitz, Shimura varieties and $\lambda$-adic representations, in Automorphic Forms, Shimura Varieties, and L-functions, New York: Academic Press (1990), Vol. 1, 161-210.

[K2] R. Kottwitz, On the $\lambda$-adic representations associated to some simple Shimura varieties, Invent. Math., 108 (1992), 653-665.

[K3] R. Kottwitz, Points on some Shimura varieties over finite fields. J. Amer. Math. Soc., 5 (1992), 373-444.

[L.IV.1] J.-P. Labesse, Changement de base CM et séries discrètes, in L. Clozel, M. Harris, J.-P. Labesse, and B. C. Ngô, eds., Stabilization of the trace formula, Shimura varieties, and arithmetic applications. Volume 1: On the stabilization of the trace formula, Boston: International Press (2011), 429-471.

[L] R. P. Langlands, Les débuts d'une formule des traces stables, Publications de l'Université Paris \%, 13 (1983).

[LR] R. P. Langlands and M. Rapoport, Shimuravarietäten und Gerben, J. reine angew. Math. (1987), 113-220.

[M] S. Morel, On the cohomology of certain noncompact Shimura varieties. With an appendix by Robert Kottwitz. Annals of Mathematics Studies, 173 (2010).

[O] T. Oda, Periods of Hilbert modular surfaces, Progress in Mathematics, 19. Birkhäuser, Boston, Mass. (1982).

[R] J. Rogawski, Automorphic representations of unitary groups in three variables, Annals of Math. Studies, 123 (1990).

[SS] P. Scholze and S. W. Shin, On the cohomology of compact unitary group Shimura varieties at ramified places, manuscript (2011).

[Se] J.-P. Serre, Sur les groupes de Galois attachés aux groupes $p$ divisibles, Proc. Conf. local fields, Driebergen, Springer-Verlag (1966), 118-131.

[S] S. W. Shin, Galois representations arising from some compact Shimura varieties, Ann. of Math., 173 (2011), 1645-1741.

[So] C. M. Sorensen, A patching lemma, manuscript at http:// fa.institut.math.jussieu.fr/node/45.

[T] R. Taylor, On Galois representations associated to Hilbert modular forms. Invent. Math., 98 (1989), 265-280. 
[TY] R. Taylor and T. Yoshida, Compatibility of local and global Langlands correspondences, J. Am. Math. Soc., 20 (2007), 467493.

[W] A. Wiles, On ordinary $\lambda$-adic representations associated to modular forms, Invent. Math., 94 (1988), 529-573.

[Wi] J-P. Wintenberger, Propriétés du groupe tannakien des structures de Hodge $p$-adiques et torseur entre cohomologies cristalline et étale, Annales de l'institut Fourier, 47 (1997), 1289-1334.

[Z] T. Zink, Über die schlechte Reduktion einiger Shimuramannigfaltigkeiten, Compositio Math., 45 (1982), 15-107.

GaËtan Chenevier

Centre de Mathématiques Laurent Schwartz

École Polytechnique

FRANCE

Michael Harris

Institut de Mathématiques de Jussieu

U.M.R. 7586 DU CNRS

FRANCE

Membre

Institut Universitaire De France

ReCEIVEd OCTOBER 18, 2011 